\title{
Constructions for t-conorms and t-norms on interval-valued and interval-valued intuitionistic fuzzy sets by paving
}

\author{
Martin Kalina \\ Department of Mathematics and Descriptive Geometry \\ Faculty of Civil Engineering, Slovak University of Technology \\ Radlinského 11, SK-810 05 Bratislava, Slovakia \\ e-mail: martin.kalina@stuba.sk
}

Received: 15 May 2020

Accepted: 16 September 2020

\begin{abstract}
Paving is a method for constructing new operations from a given one. Kalina and Král in 2015 showed that on the real unit interval this method can be used to construct associative, commutative and monotone operations from particular given operations (from basic 'paving stones'). In the present paper we modify the construction method for interval-valued fuzzy sets. From given (possibly representable) t-norms and t-conorms we construct new, non-representable operations. In the last section, we modify the presented construction method for interval-valued intuitionistic fuzzy sets.
\end{abstract}

Keywords: Interval-valued fuzzy sets, Interval-valued intuitionistic fuzzy sets, Paving, t-norm, t-conorm.

2010 Mathematics Subject Classification: 03E72, 08A72.

\section{Introduction}

Intuitionistuc fuzzy sets are a special type of lattice-valued fuzzy sets, introduced by Goguen [8]. Important mile-stones in the theory of intuitionistic fuzzy sets, besides the monograph by Atanassov [1], are the papers by Atanassov and Gargov [3], and Deschrijver et al. [7]. In [3], Atanassov and Gargov have shown that the theory of intuitionistic fuzzy sets is equivalent to the theory of interval-valued fuzzy sets. In [6,7] Deschrijver et al. have shown that there exist t-norms which are not representable as a pair of a t-norm and a t-conorm. 
In the present paper we will use the language of interval-valued fuzzy sets. A motivation for our study was the paper by Kalina and KráP [11], where the authors presented a new kind of constructions associative, commutative and monotone operations. we are going to adopt the method for interval-valued fuzzy sets. Afterwards, the results will be transformed

\section{Basic definitions and some known facts}

Interval-valued fuzzy sets (IVF-sets) are a special case of $L$-fuzzy sets [8], where $L$ is a bounded lattice. Equivalently, interval-valued fuzzy sets can be expressed as intuitionistic fuzzy sets (IF-sets) [1,3]. Membership grades of an IVF-set are elements $\left(x_{1}, x_{2}\right) \in[0,1]^{2}$ such that $x_{1} \leq x_{2}$. The set of all IVF-membership grades will be denoted by $L_{*}$. The order on $L_{*}$ is defined by

$$
\left(x_{1}, x_{2}\right) \leq_{L_{*}}\left(y_{1}, y_{2}\right) \Leftrightarrow x_{1} \leq y_{1} \& x_{2} \leq y_{2}
$$

for arbitrary $\left(x_{1}, x_{2}\right),\left(y_{1}, y_{2}\right) \in L_{*}$. Thus, the least and the greatest elements of $L_{*}$ are $\mathbf{0}=(0,0)$, $\mathbf{1}=(1,1)$, respectively. We will write elements of $L_{*}$ in bold letter to distinguish them from real numbers. Alternatively, we will use the notation $\mathbf{x}=\left(x_{1}, x_{2}\right)$, if we need to stress particular coordinates of $\mathbf{x}$. Thus we will write $L_{*}=\left\{\mathbf{x} \in[0,1]^{2} ; x_{1} \leq x_{2}\right\}$ where $\mathbf{x}=\left(x_{1}, x_{2}\right)$.

Following the notation introduced in [4], we denote $\mathbf{x} \| \mathbf{y}$ if $\mathbf{x}, \mathbf{y} \in L_{*}$ are incomparable.

\subsection{Transform between IVF-sets and IF-sets}

Membership grades of IF-sets are elements $\left(x_{1}, x_{2}\right) \in[0,1]^{2}$ such that $x_{1}+x_{2} \leq 1$. The set of all IF-membership grades will be denoted by $L^{*}$. The order on $L^{*}$ is defined by

$$
\left(x_{1}, x_{2}\right) \leq_{L^{*}}\left(y_{1}, y_{2}\right) \Leftrightarrow x_{1} \leq y_{1} \& y_{2} \geq x_{2}
$$

for arbitrary $\left(x_{1}, x_{2}\right),\left(y_{1}, y_{2}\right) \in L^{*}$. The least and the greatest elements of $L^{*}$ are $\overline{\mathbf{0}}=(0,1)$, $\overline{\mathbf{1}}=(1,0)$, respectively. The transform between $L_{*}$ and $L^{*}$ is the following $[1,3]$

$$
\text { for }\left(x_{1}, x_{2}\right) \in L_{*} \quad\left(x_{1}, 1-x_{2}\right) \text { is the corresponding element of } L^{*} .
$$

\subsection{Associative, commutative and monotone operations on $[0,1]$ and on $L_{*}$}

Associative operations such as t-norms, t-conorms, or uninorms as their common generalization, play an important role in fuzzy logic, in decision making, fuzzy control, and so on. Among these operations, those which are strictly increasing on $] 0,1\left[{ }^{2}\right.$, play an important role because of their cancelativity.

A function $N:[0,1] \rightarrow[0,1]$ is said to be a negation if $N$ is decreasing and $N(0)=1$, $N(1)=0$.

A negation $N$ is said to be strong if it is involutive, i.e., if $N(N(x))=x$ for all $x \in[0,1]$.

Definition 1. (see, e.g., $[13,15]$ ) A triangular norm $T:[0,1]^{2} \rightarrow[0,1]$ (t-norm for short) is a commutative, associative, increasing in both variables binary operation fulfilling the boundary condition $T(x, 1)=x$ for all $x \in[0,1]$. 
Remark 1. Note that, for a strong negation $N$, the $N$-dual operation to a t-norm $T$ defined by $S(x, y)=N(T(N(x), N(y)))$ is called a t-conorm. For more information, see, e.g., [13].

We will denote $T_{M}(x, y)=\min \{x, y\}$ and $S_{M}(x, y)=\max \{x, y\}$.

An operation $\tilde{T}:[0,1]^{2} \rightarrow[0,1]$ which is increasing in both variables, associative, commutative and $\tilde{T} \leq T_{M}$, is called a $t$-sub-norm.

Dually, $\tilde{S}:[0,1]^{2} \rightarrow[0,1]$ which is increasing in both variables, associative, commutative and $\tilde{S} \geq S_{M}$, is called a $t$-super-conorm.

T-sub-norms were introduced in [10] (see also [13]). T-super-conorms were introduced in [14].

Definition 2. Let $*:[0,1]^{2} \rightarrow[0,1]$ be a commutative operation. Fix a value $a \in[0,1]$. We say that $x \in[0,1], x \neq a$, is an a-divisor if there exists $y \in[0,1], y \neq a$ such that

$$
x * y=a .
$$

Further, for increasing functions $f:[a, b] \rightarrow[c, d]$, functions $f^{\wedge}:[c, d] \rightarrow[a, b]$ and $f^{\vee}:$ $[c, d] \rightarrow[a, b]$ were introduced in [12] as follows

$$
\begin{aligned}
& f^{\wedge}(y)=\sup \{z \in[a, b] ; f(z)<y\}, \\
& f^{\vee}(y)=\inf \{z \in[a, b] ; f(z)>y\},
\end{aligned}
$$

where $\sup \emptyset=a, \inf \emptyset=b$.

Definition 3 ([12]). Let $f:[a, b] \rightarrow[c, d]$ be an increasing function. An arbitrary function $f^{*}:[c, d] \rightarrow[a, b]$ is called a quasi-inverse of $f$ if it satisfies

$$
\begin{aligned}
& f\left(f^{*}(z)\right)=z \text { for } z \in \operatorname{rng}(f), \\
& f^{\wedge} \leq f^{*} \leq f^{\vee} .
\end{aligned}
$$

Remark 2. Of course, $f^{\wedge}$ coincides with the well-known pseudo-inverse $f^{(-1)}$. If $f$ is continuous with $\operatorname{rng}(f)=[c, d]$, both $f^{\wedge}$ as well as $f^{\vee}$, are special cases of quasi-inverses of $f$.

Remark 3. Assume $T_{1}, T_{2}$ are t-norms on $[0,1]$ such that $T_{1}(x, y) \leq T_{2}(x, y)$ holds for all $(x, y) \in[0,1]^{2}$. Then $\mathcal{T}: L_{*}^{2} \rightarrow L_{*}$, defined by

$$
\mathcal{T}\left(\left(x_{1}, x_{2}\right),\left(y_{1}, y_{2}\right)\right)=\left(T_{1}\left(x_{1}, y_{1}\right), T_{2}\left(x_{2}, y_{2}\right)\right),
$$

is a t-norm on $L_{*}$. Similarly we can construct a t-conorm on $L_{*}$. Such operations are called representable (see $[6,7]$ ). In $[6,7]$, Deschrijver et al. constructed also some non-representable t-norms.

Example 1 ([6]). Let $T:[0,1]^{2} \rightarrow[0,1]$ be a t-norm. Then

$$
\begin{aligned}
\mathcal{T}_{T}\left(\left(x_{1}, x_{2}\right),\left(y_{1}, y_{2}\right)\right) & =\left(T\left(x_{1}, y_{1}\right), \max \left(T\left(x_{1}, y_{2}\right), T\left(x_{2}, y_{1}\right)\right),\right. \\
\mathcal{T}_{T, t}\left(\left(x_{1}, x_{2}\right),\left(y_{1}, y_{2}\right)\right) & =\left(T\left(x_{1}, y_{1}\right), \max \left(T\left(t, T\left(x_{2}, y_{2}\right), T\left(x_{1}, y_{2}\right), T\left(x_{2}, y_{1}\right)\right)\right.\right.
\end{aligned}
$$

for arbitrarily chosen $t \in[0,1]$, are t-norms on $L_{*}$. The t-norm $\mathcal{T}_{T}$ is called pseudo-t-representable, the t-norm $\mathcal{T}_{T, t}$ is called generalized pseudo-t-representable.

For more details on t-norms and t-conorms we recommend the monographs [13,15]. For other types of aggregation functions we recommend the monograph [9]. 


\subsection{Paving as construction method for operations on $[0,1]$}

The idea of paving (see [11], for further modifications [5] and [16]) is the following. We split the unit interval into countably many disjoint subintervals $\left\{I_{i}\right\}_{i \in \mathcal{I}}$ (in such a way we split the unit square into countably many disjoint sub-rectangles $I_{i} \times I_{j}$ ). Then we take an operation $*:[0,1]^{2} \rightarrow[0,1]$, choose transformations $f_{i}: I_{i} \rightarrow[0,1]$ and we 'pave' the whole unit square (see Fig. 1 for a graphical schema of paving) by

$$
f_{i+j}^{[-1]}\left(f_{i}(x) * f_{j}(y)\right)
$$

where $f_{i+j}^{[-1]}$ is a kind of inverse (could be pseudo-inverse or a quasi-inverse).

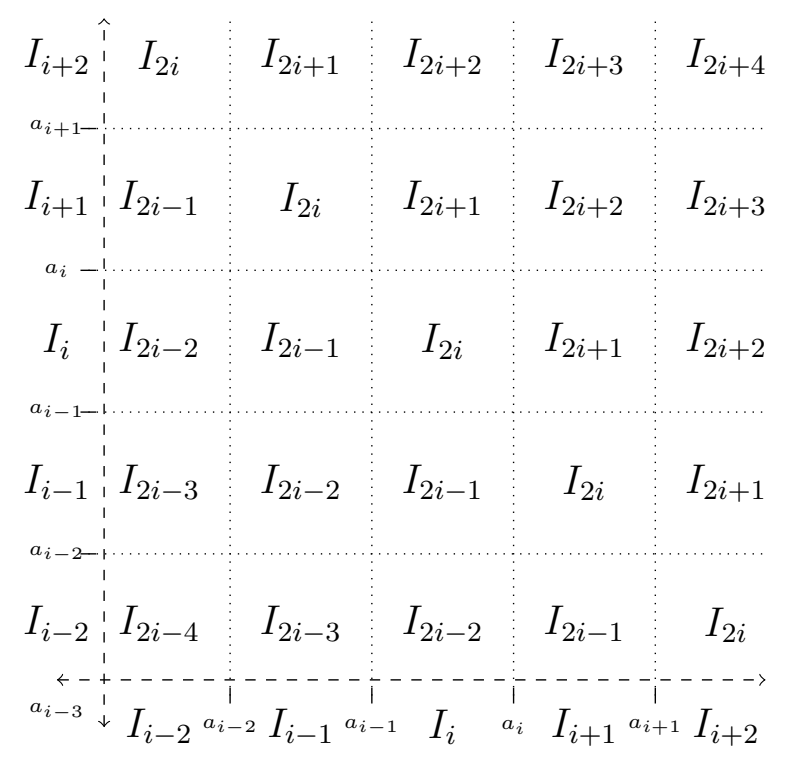

Figure 1. Graphical schema of paving

Our intention is to construct an operation $\odot:[0,1]^{2} \rightarrow[0,1]$ which is (not necessarily strictly) increasing in both variables, commutative and associative. We split the unit interval $[0,1]$ into countably many sub-intervals by choosing a sequence of their end-points. For a technical reason we will need the set of indices to be closed under addition (or by any other associative, commutative and monotone operation). The set of indices will be denoted by $\mathcal{J}$. We set

$$
0=a_{0}<a_{1}<a_{2}<\cdots<a_{n}<\cdots<a_{\infty}=1
$$

For each interval $I_{i}=\left[a_{i-1}, a_{i}\right]$ we need a monotone transformation $f_{i}: I_{i} \rightarrow[0,1]$. We assume $f_{i}\left(a_{i-1}\right)=0$ and $f_{i}\left(a_{i}\right)=1$. Moreover, we will assume continuity of all functions $f_{i}$.

Because of uniqueness of the assignment we consider semi-open intervals. We introduce the following notation:

$$
I_{i}^{<}=\left[a_{i-1}, a_{i}\left[, \quad I_{i}^{>}=\right] a_{i-1}, a_{i}\right] .
$$

The basic idea of paving is hidden in the following two formulae which will be used alternatively. We choose an operation $*:[0,1]^{2} \rightarrow[0,1]$ which is isotone in both variables, commutative 
and associative. Then, for $i, j \in \mathcal{J}, x \in I_{i}^{<}$and $y \in I_{j}^{<}\left(x \in I_{i}^{>}\right.$and $y \in I_{j}^{>}$, alternatively) we define

$$
x *^{\vee} y=f_{i+j}^{\vee}\left(f_{i}(x) * f_{j}(y)\right)
$$

and

$$
x *^{\wedge} y=f_{i+j}^{\wedge}\left(f_{i}(x) * f_{j}(y)\right) .
$$

Formulae (8) and (9) directly imply that both operations, $*^{\vee}$ and $*^{\wedge}$, are isotone and commutative (when properly defined on the border).

We know already that, for continuous $f_{i}$ we have $f_{i}\left(f_{i}^{\vee}(x)\right)=x$ as well as $f_{i}\left(f_{i}^{\wedge}(x)\right)=x$. This means that if we use left-closed intervals, the operation $*^{\vee}$ is associative if

$$
f_{i+j}^{\vee}\left(f_{i}(x) * f_{j}(y)\right) \in I_{i+j}^{<}
$$

for all $x \in I_{i}^{<}$and $y \in I_{j}^{<}$, and the same condition has to be fulfilled also for the operation $*^{\wedge}$ (and analogically we could treat the situation with right-closed intervals). The reader can find more details in [11].

\section{Modification of paving for IVF-sets}

When considering IVF-sets instead of the unit real interval (or a chain, in general) we have to overcome a new technical problem. Namely, on IVF-sets there are incomparable elements. We must pay attention to keeping monotonicity. We split the triangular area of $L_{*}$ as indicated in Fig. 2 into infinitely many rectangular and infinitely many triangular areas $I_{(i, j)}$ where

(A) $j \in\{0,1,2 \ldots, n, \ldots\}, i \in\{0,1,2, \ldots, j\}$.

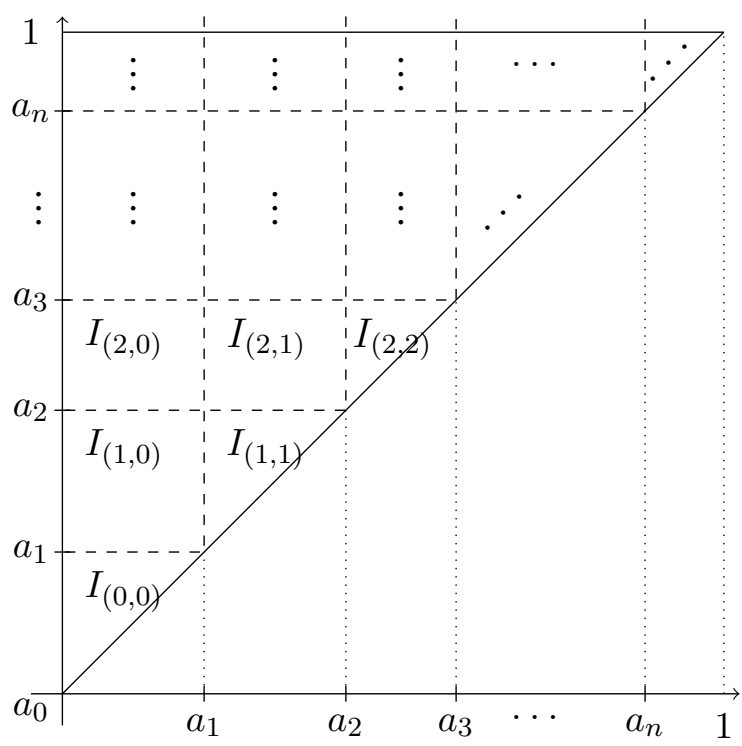

Figure 2. Graphical schema of splitting $L_{*}$ into rectangular and triangular areas

To preserve uniqueness of the partition of $L_{*}$, we have two possibilities concerning the edges of rectangular and triangular areas: 
1. We remove the upper and right edges from all rectangular areas and the upper edge from all triangular areas, these types of areas will be denoted $I_{(i, j)}^{<}$, i.e., for rectangular areas $I_{(i, j)}^{<}=\left[a_{i}, a_{i+1}\left[\times\left[a_{j}, a_{a+1}[\right.\right.\right.$,

2. We remove the the lower and left edges from all rectangular areas and the left edge from all triangular areas, these types of areas will be denoted $I_{(i, j)}^{>}$, i.e., for recangular areas $\left.\left.\left.\left.I_{(i, j)}^{<}=\right] a_{i}, a_{i+1}\right] \times\right] a_{j}, a_{a+1}\right]$.

Now, we choose transforms of the rectangular and triangular areas into $L_{*}$. We set transforms $\varrho_{(i, j)}: I_{(i, j)} \rightarrow L_{*}, i<j$, for the rectangular, and $\delta_{i}: I_{(i . i)} \rightarrow L_{*}$ for the triangular areas. Now, we define transforms for $I_{(i, j)}$ including all edges.

$$
\begin{aligned}
\varrho_{(i, j)}(\mathbf{x}) & =\left(\frac{x_{1}-a_{i}}{a_{i+1}-a_{i}} ; \frac{x_{2}-a_{j}}{a_{j+1}-a_{j}} \cdot \frac{a_{i+1}-x_{1}}{a_{i+1}-a_{i}}+\frac{x_{1}-a_{i}}{a_{i+1}-a_{i}}\right), \\
\delta_{i}(\mathbf{x}) & =\left(\frac{x_{1}-a_{i}}{a_{i+1}-a_{i}} ; \frac{x_{2}-a_{i}}{a_{i+1}-a_{i}}\right) .
\end{aligned}
$$

Alternatively, instead of the transforms $\varrho_{(i, j)}$ we can use $\bar{\varrho}_{(i, j)}$ :

$$
\bar{\varrho}_{(i, j)}(\mathbf{x})=\left(\frac{x_{1}-a_{i}}{a_{i+1}-a_{i}} \cdot \frac{x_{2}-a_{j}}{a_{j+1}-a_{j}} ; \frac{x_{2}-a_{j}}{a_{j+1}-a_{j}}\right) .
$$

What happens to the coordinates of $\mathbf{x}=\left(x_{1}, x_{2}\right)$ when using particular transforms, is the following:

1. We shift the area so that the left down vertex $\left(a_{i}, a_{j}\right)$ moved to $(0,0)$, we get $I_{(i, j)}^{1}$

2. We extend $I_{(i, j)}^{1}$ to have $\left(a_{i}, a_{j+1}\right) \mapsto(0,1),\left(a_{i+1}, a_{j+1}\right) \mapsto(1,1)$. Now, rectangular areas are transformed to $[0,1]^{2}$ (denoted by $I_{(i, j)}^{2}$ ) and triangular to $L_{*}$.

3. When using $\varrho_{(i, j)}$, we transform the second coordinate in $I_{(i, j)}^{2}$ so that the cut with the first coordinate $\tilde{x}_{1}$ is shrinked to $1-\tilde{x}_{1}$, and then is moved upwards $\tilde{x}_{1}$.

When using $\bar{\varrho}_{(i, j)}$, we transfor the first coordinate in $I_{(i, j)}^{2}$ so that the cut with the second coordinate $\tilde{x}_{2}$ is shrinked to $\tilde{x}_{2}$.

Hence, we get that, when using transforms $\varrho_{(i, j)}$, the right edges of rectangular areas are transformed to $(1,1)$, when using transforms $\bar{\varrho}_{(i, j)}$, the lower edges are transformed to $(0,0)$. All other points are in one-one correspondence.

This reasoning leads to the conclusion that, for $I_{(i, j)}^{<}$transforms $\varrho_{(i, j)}$ are suitable and for $I_{(i, j)}^{>}$ transforms $\bar{\varrho}_{(i, j)}$ are suitable.

\subsection{Construction of new t-conorms from a given one}

In this part we describe in details the construction of a t-conorm by paving for the two mentioned possibilities, namely, using $I_{(i, j)}^{<}$and $I_{(i, j)}^{>}$areas. 
Proposition 1. Let $\mathcal{S}: L_{*}^{2} \rightarrow L_{*}$ be a t-conorm such that, for all $\mathbf{x}, \mathbf{y} \in L_{*}$ with $x_{2}<1$ and $y_{2}<1$, we have $\mathcal{S}(\mathbf{x}, \mathbf{y})=\left(z_{1}, z_{2}\right)$ where $z_{2}<1$. Denote $\sum_{\mathbf{x}, \mathbf{y}}=i+j+k+\ell$ for $(\mathbf{x}, \mathbf{y}) \in I_{i, j} \times I_{k, \ell}$ and

$$
\Theta_{(i, j)}(\mathbf{x})=\left\{\begin{array}{lll}
\varrho_{(i, j)}(\mathbf{x}) & \text { if } & i<j, \\
\delta_{i}(\mathbf{x}) & \text { if } & i=j .
\end{array}\right.
$$

Then the following function is a t-conorm

$$
\mathbb{S}_{1}(\mathbf{x}, \mathbf{y})= \begin{cases}\mathbf{x} \vee \mathbf{y} & \text { if } \mathbf{x} \wedge \mathbf{y}=\mathbf{0}, \\ \mathbf{1} & \text { if } \mathbf{x} \wedge \mathbf{y} \neq \mathbf{0} \text { and } \mathbf{x} \vee \mathbf{y} \geq(0,1), \\ \delta_{\sum_{\mathbf{x}, \mathbf{y}}}^{-1}\left(\mathcal{S}\left(\Theta_{(i, j)}(\mathbf{x}), \Theta_{(k, \ell)}(\mathbf{y})\right)\right) & \text { if }(\mathbf{x}, \mathbf{y}) \in I_{(i, j)}^{<} \times I_{(k, \ell)}^{<}, \mathbf{x} \wedge \mathbf{y} \neq \mathbf{0}\end{cases}
$$

Proof. First, we show the monotonicity of $\mathbf{S}_{1}$. We have $\mathcal{S}(\mathbf{x}, \mathbf{y}) \geq\left(\max \left(x_{1}, y_{1}\right), \max \left(x_{2}, y_{2}\right)\right)$. The only case we have to consider is when $\mathbf{x} \in I_{(0,0)}^{<}$and $y \in I_{(0, \ell)}^{<}$, or $\mathbf{y} \in I_{(0,0)}^{<}$and $x \in I_{(0, j)}^{<}$. In all other cases the monotonicity is given by the choice of the triangular area $I_{\left(\sum_{\mathbf{x}, \mathbf{y}}, \sum_{\mathbf{x}, \mathbf{y}}\right)}^{<}$into which the result is transformed.

Let $\mathbf{x} \in I_{(0,0)}^{<}$and $y \in I_{(0, \ell)}^{<}$. For keeping the monotonicity, the second coordinates are important. We have $\delta_{\ell}^{-1}\left(z_{1}, z_{2}\right)=\left(\left(a_{\ell+1}-a_{\ell}\right) z_{1}+a_{\ell},\left(a_{\ell+1}-a_{\ell}\right) z_{2}+a_{\ell}\right)$. Then we get the following lower bound for the second coordinate of $\mathbb{S}_{1}$

$$
\begin{aligned}
& \left(a_{\ell+1}-a_{\ell}\right) \max \left(\frac{x_{2}}{a_{1}}, \frac{y_{2}-a_{\ell}}{a_{\ell+1}-a_{\ell}} \cdot \frac{a_{1}-y_{1}}{a_{1}}+\frac{y_{1}}{a_{1}}\right)+a_{\ell} \geq \\
\geq & \left(y_{2}-a_{\ell}\right) \cdot \frac{a_{1}-y_{1}}{a_{1}}+\frac{y_{1}}{a_{1}} \cdot\left(a_{\ell+1}-a_{\ell}\right)+a_{\ell}=y_{2}+\frac{y_{1}}{a_{1}}\left(a_{\ell+1}-y_{2}\right) \geq y_{2}
\end{aligned}
$$

since $a_{\ell} \leq y_{2} \leq a_{\ell+1}$.

To prove the associativity, we have $\mathbb{S}_{1}\left(\mathbf{x}, \mathbb{S}_{1}(\mathbf{y}, \mathbf{z})\right)=\mathbb{S}_{1}\left(\mathbb{S}_{1}(\mathbf{x}, \mathbf{y}), \mathbf{z}\right)=\mathbf{1}$ if and only if $\max \left(x_{2}, y_{2}, z_{2}\right)=1$.

If $\max \left(x_{2}, y_{2}, z_{2}\right)<1$ and all $\mathbf{x}, \mathbf{y}, \mathbf{z}$, are different form $\mathbf{0}$, we have

$$
\mathbb{S}_{1}(\mathbf{x}, \mathbf{y}) \in I_{\left(\sum_{\mathbf{x}, \mathbf{y}}, \sum_{\mathbf{x}, \mathbf{y}}\right)}^{<} \quad \text { for } \quad \mathbf{x} \in I_{(i, j)}^{<} \text {and } \mathbf{y} \in I_{(k, \ell)}^{<}
$$

and in that case, when computing $\mathbb{S}_{1}\left(\mathbb{S}_{1}(\mathbf{x}, \mathbf{y}), \mathbf{z}\right), \delta_{\sum_{\mathbf{x}, \mathbf{y}}}^{-1}$ is cancelled. This implies that the associativity of $\mathbb{S}_{1}$ is a concequence of the associativity of $\mathcal{S}$

A new t-conorm can be constructed for a given one also when using areas of the type $I_{i, j}^{>}$.

Proposition 2. Let $\mathcal{S}: L_{*}^{2} \rightarrow L_{*}$ be an arbitrary $t$-conorm. Denote $\sum_{\mathbf{x}, \mathbf{y}}=i+j+k+\ell$ for $(\mathbf{x}, \mathbf{y}) \in I_{(i-j)} \times I_{(k, \ell)}$ and

$$
\Psi_{(i, j)}(\mathbf{x})=\left\{\begin{array}{lll}
\bar{\varrho}_{(i, j)}(\mathbf{x}) & \text { if } & i<j, \\
\delta_{i}(\mathbf{x}) & \text { if } & i=j .
\end{array}\right.
$$

Then the following function is a t-conorm

$$
\mathbb{S}_{2}(\mathbf{x}, \mathbf{y})= \begin{cases}\mathbf{1} & \text { if } \mathbf{x} \vee \mathbf{y} \geq(0,1), \\ \mathbf{x} \vee \mathbf{y} & \text { if } \mathbf{x} \wedge \mathbf{y} \leq(0,1) \text { and } \mathbf{x} \vee \mathbf{y} \geq(0,1), \\ \delta_{\sum_{\mathbf{x}, \mathbf{y}}}^{-1}\left(\mathcal{S}\left(\Psi_{(i, j)}(\mathbf{x}), \Psi_{(k, \ell)}(\mathbf{y})\right)\right. & \text { for }(\mathbf{x}, \mathbf{y}) \in I_{(i, j)}^{>} \times I_{(k, \ell)}^{>} .\end{cases}
$$


Proof. We give just an idea of the proof since it follows a similar idea as that of Proposition 1. For the monotonicity just realize that, when $\mathbf{x} \in I_{(0,0)}^{>}$and $\mathbf{y} \in I_{(0, \ell)}^{>}$, the second coordinate of $\delta_{\ell}^{-1}$ is just the inverse transformation for the second coordinate of $\bar{\varrho}_{(0, \ell)}$. In all other cases the monotonicity is directly due to the construction of $\mathbb{S}_{2}$.

For the associativity we can just re-write the proof of Proposition 1.

For a better understanding how the paving works, we sketch Fig. 3. The areas with equal shade of gray have values from the same triangular area. The darker the shade, the larger the values. This means, the values increase diagonally.

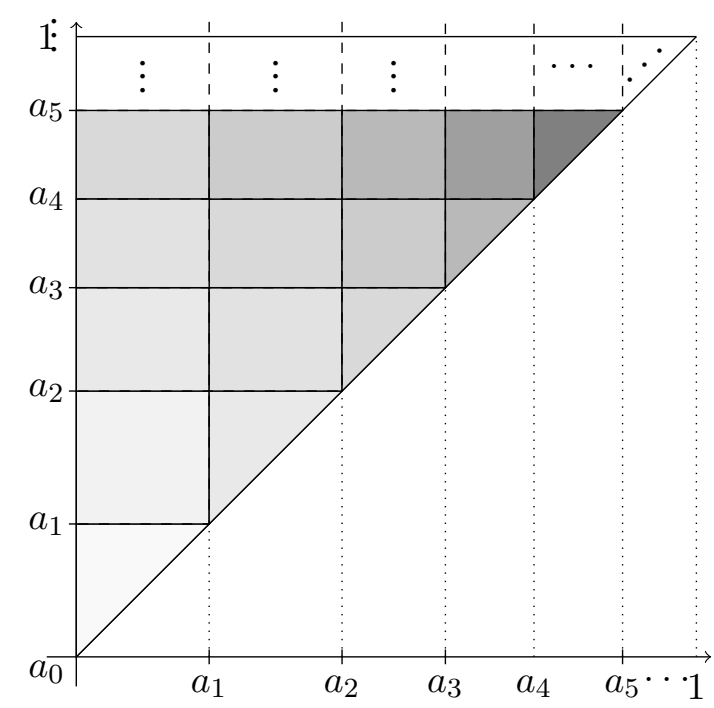

Figure 3. Graphical schema of paving on $L_{*}$

Remark 4. Since for every t-super-conorm $\tilde{\mathcal{S}}(\mathbf{x}, \mathbf{y}) \geq \mathbf{x} \vee \mathbf{y}$ holds, in Propositions 1 and 2 we can exchange the t-conorm $\mathcal{S}$ for a t-super-conorm $\tilde{\mathcal{S}}$. The resulting functions $\tilde{\mathbb{S}}_{1}$ and $\tilde{\mathbb{S}}_{2}$ are again t-conorms if $\tilde{\mathcal{S}}$ yield the same constraints as the t-conorms $\mathcal{S}$ in respective Propositions 1 and 2.

\subsection{Other possibilities for construction of new t-conorms}

The t-conorms constructed in Propositions 1 and 2 and also their modifications mentioned in Remark 4 have been constructed from some t-conorms $\mathcal{S}$ or t-super-conorms $\tilde{\mathcal{S}}$, respectively (mentioned in Remark 4). These operations have one important property, namely $\mathcal{S}(\mathbf{x}, \mathbf{y}) \geq \mathbf{x} \vee \mathbf{y}$ and also $\tilde{\mathcal{S}}(\mathbf{x}, \mathbf{y}) \geq \mathbf{x} \vee \mathbf{y}$. All other binary functions are either below or incomparable with the lattice disjunction. For this reason, for the next constructions of t-conorms, we change the enumeration of the splitting $L_{*}$ into $\left\{I_{(i, j)}\right\}_{i \in \mathcal{I}, j \in \mathcal{J}}$ for the index-sets

(B) $\mathcal{J}=\{1,2,3, \ldots, n, \ldots\}, \mathcal{I}=\{1,2, \ldots, j\}$.

We will refer to this enumeration as of type (B).

Proposition 3. Let $\mathcal{V}: L_{*}^{2} \rightarrow L_{*}$ be an arbitrary associative, commutative and increasing binary function. Let us have families of areas $\mathcal{I}^{<}=\left\{I_{(i, j)}^{<}\right\}_{i \in \mathcal{I}, j \in \mathcal{J}}$ and $\mathcal{I}^{>}=\left\{I_{(i, j)}^{>}\right\}_{i \in \mathcal{I}, j \in \mathcal{J}}$ and the 
enumeration of type $(B)$. Denote $\Theta_{(i . j)}$ and $\Psi_{(i, j)}$ the transforms of $I_{(i, j)}$ defined by formulae (13) and (15), respectively. Denote $\sum_{\mathbf{x}, \mathbf{y}}=i+j+k+\ell$ for $(\mathbf{x}, \mathbf{y}) \in I_{(i, j)} \times I_{(k, \ell)}$. Assume $\mathcal{V}$ be such that $\mathcal{V}(\mathbf{x}, \mathbf{y})=\left(z_{1}, z_{2}\right)$ with $z_{2} \neq 1$ whenever $x_{2} \neq 1$ and $y_{2} \neq 1$. Then the following function is a t-conorm

$$
\mathbb{S}_{3}(\mathbf{x}, \mathbf{y})= \begin{cases}\mathbf{x} \vee \mathbf{y} & \text { if } \mathbf{x} \wedge \mathbf{y}=\mathbf{0}, \\ \mathbf{1} & \text { if } \mathbf{x} \wedge \mathbf{y} \neq \mathbf{0} \text { and } \mathbf{x} \vee \mathbf{y} \geq(0,1), \\ \delta_{\sum_{\mathbf{x}, \mathbf{y}}}^{-1}\left(\mathcal{V}\left(\Theta_{(i, j)}(\mathbf{x}), \Theta_{(k, \ell)}(\mathbf{y})\right)\right) & \text { if }(\mathbf{x}, \mathbf{y}) \in I_{(i, j)}^{<} \times I_{(k, \ell)}^{<}, \mathbf{x} \wedge \mathbf{y} \neq \mathbf{0}\end{cases}
$$

Assume $\mathcal{V}$ be such that $\mathcal{V}(\mathbf{x}, \mathbf{y})=\left(z_{1}, z_{2}\right)$ with $z_{1} \neq 0$ whenever $x_{1} \neq 0$ and $y_{1} \neq 0$. Then the following function is a $t$-conorm

$$
\mathbb{S}_{4}(\mathbf{x}, \mathbf{y})= \begin{cases}\mathbf{1} & \text { if } \mathbf{x} \vee \mathbf{y} \geq(0,1), \\ \mathbf{x} \vee \mathbf{y} & \text { if } \mathbf{x} \wedge \mathbf{y} \leq(0,1) \text { and } \mathbf{x} \vee \mathbf{y} \geq(0,1), \\ \delta_{\sum_{\mathbf{x}, \mathbf{y}}}^{-1}\left(\mathcal{V}\left(\Psi_{(i, j)}(\mathbf{x}), \Psi_{(k, \ell)}(\mathbf{y})\right)\right. & \text { for }(\mathbf{x}, \mathbf{y}) \in I_{(i, j)}^{>} \times I_{(k, \ell)}^{>} .\end{cases}
$$

Proof. The constructions of $\mathbb{S}_{3}$ and $\mathbb{S}_{4}$ are modifications of $\mathbb{S}_{1}$ and $\mathbb{S}_{2}$, introduced by (14) and (16), respectively. Just in the cases of $\mathbb{S}_{3}$ and $\mathbb{S}_{4}$ we use a general binary increasing associative and commutative function $\mathcal{V}$. This means, we have to check the monotonicity of the resulting functions. Since by the enumeration of type (B) we have $\sum_{\mathbf{x}, \mathbf{y}}>\max (i, j, k, \ell)$ and moreover,

$$
\begin{gathered}
\delta_{\sum_{\mathbf{x}, \mathbf{y}}}^{-1}\left(\mathcal{V}\left(\Theta_{(i, j)}(\mathbf{x}), \Theta_{(k, \ell)}(\mathbf{y})\right)\right) \in I_{\left(\sum_{\mathbf{x}, \mathbf{y}}, \sum_{\mathbf{x}, \mathbf{y}}\right)}^{<}, \\
\delta_{\sum_{\mathbf{x}, \mathbf{y}}}^{-1}\left(\mathcal{V}\left(\Psi_{(i, j)}(\mathbf{x}), \Psi_{(k, \ell)}(\mathbf{y})\right) \in I_{\left(\sum_{\mathbf{x}, \mathbf{y}}, \sum_{\mathbf{x}, \mathbf{y}}\right)}^{>},\right.
\end{gathered}
$$

the monotonicity is guaranteed.

\subsection{Some modifications of paving}

Other possibilities for constructing new t-conorms we get when splitting the lattice $L_{*}$ into finitely

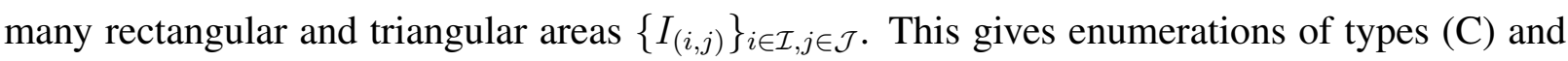
(D):

(C) $\mathcal{J}=\{0,1,2, \ldots, n\}, \mathcal{I}=\{0,1,2, \ldots, j\}$,

(D) $\mathcal{J}=\{1,2, \ldots, n\}, \mathcal{I}=\{1,2, \ldots, j\}$.

In these cases, formulae (14), (16), (17) and (18) remain valid with one change, namely, if $\sum_{\mathbf{x}, \mathbf{y}} \geq n$ in expressions $\delta_{\sum_{\mathbf{x}, \mathbf{y}}}^{-1}\left(\mathcal{V}\left(\Theta_{(i, j)}(\mathbf{x}), \Theta_{(k, \ell)}(\mathbf{y})\right)\right)$ or $\delta_{\sum_{\mathbf{x}, \mathbf{y}}}^{-1}\left(\mathcal{V}\left(\Psi_{(i, j)}(\mathbf{x}), \Psi_{(k, \ell)}(\mathbf{y})\right)\right.$ we set $\delta_{\sum_{\mathbf{x}, \mathbf{y}}}^{-1}=\mathbf{1}$.

Another possibility to modify the constructions is to change addition for computing the new index in $\sum_{\mathbf{x}, \mathbf{y}}$ for another operation $\otimes$ that is strictly increasing, associative and commutative, e.g., for multiplication. When using $\otimes$, we have to modify the index-set $\mathcal{J}$ (see $[5,16]$ such that

$$
j_{1} \otimes j_{2} \in \mathcal{J} \quad \text { for all } j_{1}, j_{2} \in \mathcal{J} \text {. }
$$

When constructing t-norms, we can just use the duality between t-norms and t-conorms. 


\section{A note on the transform of the results into interval-valued IF-sets}

All the results achieved in this paper for IVF-sets are directly transformable into IF-sets just starting with an IF-operation and changing the enumeration of splitting $[0,1]$ in the second coordinate into

$$
1=a_{0}>a_{1}>a_{2}>\cdots>a_{n}>\cdots>a_{\infty}=0 .
$$

Interval-valued intuitionistic fuzzy sets (IVIF-sets) were introduced in [3] and are well described in the recent monograph [2]. IVIF-sets are a generalization of the IF-sets. This means, they are also a special kind of lattice-valued fuzzy sets [8]. The membership grades of IVIF-sets are given by quadruples $\left(x_{1}, x_{2}, x_{3}, x_{4}\right) \in[0,1]^{4}$ such that

$$
x_{1} \leq x_{2} \& x_{3} \leq x_{4} \& x_{2}+x_{4} \leq 1 .
$$

The interval $\left[x_{1}, x_{2}\right]$ represents the membership of an element $\xi$ of a universe and the interval $\left[x_{3}, x_{4}\right]$ represents the non-membership of $\xi$. The set of all membership grades of IVIF-sets will be denoted by $L^{[*]}$. The order of elements in $L^{[*]}$ is given by

$$
\left(x_{1}, x_{2}, x_{3}, x_{4}\right) \leq_{L^{[*]}}\left(y_{1}, y_{2} \cdot y_{3} \cdot y_{4}\right) \Leftrightarrow x_{1} \leq y_{1} \& x_{2} \leq y_{2} \& x_{3} \geq y_{3} \& x_{4} \geq y_{4} .
$$

The least and the greatest elements of $L^{[*]}$ are $\mathbf{0}_{L^{[*]}}=(0,0,1,1)$ and $\mathbf{1}_{L^{[*]}}=(1,1,0,0)$, respectively.

There are several possibilities how to adopt the construction for $\mathrm{t}$-conorms (t-norms) by paving on IVIF-sets. We show now one of the constructions for t-conorms.

For every coordinate we split the interval $[0,1]$ into infinitely many disjoint subintervals choosing nods in the following way:

- for the first and the second coordinate

$$
0=a_{0}<a_{1}<a_{2}<\cdots<a_{n}<\cdots<a_{\infty}=1
$$

- for the third and the fourth coordinate

$$
1=a_{0}>a_{1}>a_{2}>\cdots>a_{n}>\cdots>a_{\infty}=0 .
$$

$$
\begin{array}{ll}
\text { Thus, for }\left(x_{1}, x_{2}, x_{3}, x_{4}\right) \in L^{[*]}, & a_{j} \leq x_{i}<a_{j+1} \Rightarrow x_{i} \in I_{j} \text { if } i \in\{1,2\}, \\
& a_{j} \geq x_{i}>a_{j+1} \Rightarrow x_{i} \in I_{j} \text { if } i \in\{3,4\} .
\end{array}
$$

We will denote $\left(x_{1}, x_{2}, x_{3}, x_{4}\right) \in I_{i_{1}, i_{2}, i_{3}, i_{4}}$ for $x_{k} \in I_{k}, k \in\{1,2,3,4\}$.

Proposition 4. Let $\mathcal{S}: L^{[*]} \times L^{[*]} \rightarrow L^{[*]}$ be a t-conorm such that, for all $\mathbf{x}, \mathbf{y} \in L^{[*]}$ with for $x_{2}<1$ and $y_{2}<1$, we have $\mathcal{S}(\mathbf{x}, \mathbf{y})=\left(z_{1}, z_{2}, z_{3}, z_{4}\right)$ where $z_{2}<1$, and if $x_{3}>0$ and $y_{3}>0$ also $z_{3}>0$. For $(\mathbf{x}, \mathbf{y}) \in I_{i_{1}, i_{2}, i_{3}, i_{4}} \times I_{j_{1}, j_{2}, j_{3}, j_{4}}$ denote

$$
\begin{aligned}
& \sum_{\mathbf{x}, \mathbf{y}}^{1}=i_{1}+i_{2}+i_{4}+j_{1}+j_{2}+j_{4}, \\
& \sum_{\mathbf{x}, \mathbf{y}}^{2}=i_{1}+i_{2}+j_{1}+j_{2}, \\
& \sum_{\mathbf{x}, \mathbf{y}}^{3}=i_{1}+i_{2}+i_{3}+i_{4}+j_{1}+j_{2}+j_{3}+j_{4}
\end{aligned}
$$


and, for $\left(x_{k}, x_{\ell}\right)$ where $(k, \ell) \in\{(1,2),(2,4),(3,4)\}, x_{k} \in I_{i}, x_{\ell} \in I_{j}$, denote

$$
\Theta_{(i, j)}\left(x_{k}, x_{\ell}\right)=\left\{\begin{array}{lll}
\varrho_{(i, j)}\left(x_{k}, x_{\ell}\right) & \text { if } & i<j, \\
\delta_{i}\left(x_{k}, x_{\ell}\right) & \text { if } i=j,
\end{array}\right.
$$

where $\varrho$ and $\delta$ are defined by (10) and (11), respectively. Then the following function is a t-conorm

$$
\mathbb{S}_{I V I F}(\mathbf{x}, \mathbf{y})= \begin{cases}\mathbf{x} \vee \mathbf{y} & \text { if } \mathbf{x} \wedge \mathbf{y}=\mathbf{0}, \\ \mathbf{1}_{L^{[*]}} & \text { if } \mathbf{x} \wedge \mathbf{y} \neq \mathbf{0} \text { and } x_{4} \wedge y_{4}=0, \\ \left(z_{1}, z_{2}, z_{3}, z_{4}\right) & \text { otherwise, }\end{cases}
$$

where

$$
\begin{aligned}
z_{1} & =\Pi_{1}\left(\delta_{\sum_{\mathbf{x}, \mathbf{y}}^{2}}^{-1}\left(\mathcal{S}_{(1,2)}\left(\Theta_{\left(i_{1}, i_{2}\right)}\left(x_{1}, x_{2}\right), \Theta_{\left(j_{1}, j_{2}\right)}\left(y_{1}, y_{2}\right)\right)\right),\right. \\
\left(z_{2}, z_{4}\right) & =\delta_{\sum_{\mathbf{x}, \mathbf{y}}^{-1}}^{-1}\left(\mathcal{S}_{(2,4)}\left(\Theta_{\left(i_{2}, i_{4}\right)}\left(x_{2}, x_{4}\right), \Theta_{\left(j_{2}, j_{4}\right)}\left(y_{2}, y_{4}\right)\right),\right. \\
z_{3} & =\Pi_{1}\left(\delta_{\sum_{\mathbf{x}, \mathbf{y}}^{3}}^{-1}\left(\mathcal{S}_{(3,4)}\left(\Theta_{(i, j)}\left(x_{3}, x_{4}\right), \Theta_{(k, \ell)}\left(y_{3}, y_{4}\right)\right)\right)\right),
\end{aligned}
$$

and $\Pi_{1}$ means the projection into the first coordinate and $\mathcal{S}_{(a, j)}$ means the $i$-th and the $j$-th coordinate of $\mathcal{S}$.

The proof of Proposition 4 is just a modification of the proof of Proposition 1. That is why it is omitted.

\section{Conclusions}

We discussed some construction possibilities for t-conorms by paving. Particularly, we provided explicitly four formulea showing the construction of new t-conorms from given increasing associative and commutative operations. Finally, we gave some modifications of these construction possibilities. All the constructed t-conorms are non-representable in the sence of $[6,7]$. New t-norms can be constructed just by their duality with t-conorms. In Section 4, first, we made a comment on how the results for interval-valued fuzzy sets can be transformed to intuitionistic fuzzy sets. Finally, we extendeded the results to the case of interval-valued intuitionistic fuzzy sets where, in Proposition 4, we constructed a t-conorm by paving.

\section{Acknowledgements}

The work on this paper was supported from the Science and Technology Assistance Agency under the contract no. APVV-18-0052, and by the Slovak Scientific Grant Agency VEGA, grant no. $1 / 0006 / 19$ and $2 / 0142 / 20$. 


\section{References}

[1] Atanassov, K. T. (1999). Intuitionistic Fuzzy Sets: Theory and Applications. Springer, Heidelberg.

[2] Atanassov, K. T. (2020). Interval-Valued Intuitionistic Fuzzy Sets. Springer, Cham.

[3] Atanassov, K., \& Gargov, G. (1989). Interval valued intuitionistic fuzzy sets, Fuzzy Sets and Systems, 31 (3), 343-349.

[4] Birkhoff, G. (1967). Lattice Theory. American Mathematical Society Colloquium Publishers, Providence, RI.

[5] Bodjanova, S., \& Kalina, M. (2017). Block-wise construction of commutative increasing monoids, Fuzzy Sets and Systems, 324, 91-99.

[6] Deschrijver, G. (2008). A representation of t-norms in interval valued L-fuzzy set theory, Fuzzy Sets and Systems, 159, 1597-1618.

[7] Deschrijver, G., Cornelis, C., \& Kerre, E. E. (2004). On the representation of intuitionistic fuzzy t-norms and t-conorms, IEEE Trans. Fuzzy Systems, 12 (1), 45-61.

[8] Goguen, J. A. (1967). L-fuzzy sets. Journal of Mathematical Analysis and Applications, $18(1), 145-174$.

[9] Grabisch, M., Marichal, J. L., Mesiar, R., \& Pap, E. (2009). Aggregation Functions, Cambridge University Press, Cambridge.

[10] Jenei, S. (2001). Structure of left-continuous triangular norms with strong induced negations, (II) Rotation-annihilation construction, Journal of Applied Non-Classical Logic, $11,351-366$.

[11] Kalina, M., \& Král, P. (2015). Construction of commutative associative operations by paving. In: Alonso, J. M., Bustince, H., Reformat M. (eds.) IFSA-EUSFLAT 2015, (Atlantis Press, Gijón, 2015), 1201-1207.

[12] Klement, E. P., Mesiar, R., \& Pap, E. (1999). Quasi- and pseudo-inverses of monotone functions, and the construction of t-norm, Fuzzy Sets and Systems, 104, 3-13.

[13] Klement, E.P., Mesiar, R., \& Pap, E. (2000). Triangular Norms. Springer, Berlin, Heidelberg.

[14] Mesiarová-Zemánková, A. (2016). Ordinal sum construction for uninorms and generalized uninorms International Journal of Approximate Reasoning, 76, 1-17.

[15] Schweizer B., \& Sklar, A. (1983) Probabilistic Metric Spaces, North Holland, New York.

[16] Zong, W., Su, Y., Liu, H. W., \& De Baets, B. (2017) On the Construction of Associative, Commutative and Increasing Operations by Paving, in Torra V., Mesiar R., Baets B. (eds) Aggregation Functions in Theory and in Practice. AGOP 2017. Advances in Intelligent Systems and Computing, vol 581, Springer, Cham, 229-240. 